\title{
TWO-TIMING PROCEDURE FOR HIGHER-ORDER MODULATIONS OF NEAR-LINEAR DISPERSIVE WAVE TRAINS WITH AN APPLICATION TO PLASMA WAVES*
}

\author{
BY \\ P. GATIGNOL \\ Université Pierre et Marie Curie, Paris
}

\begin{abstract}
An asymptotic procedure is proposed to describe the slow modulations of dispersive wave trains when the dispersive effects and the nonlinear distorsion are of the same order of magnitude. For a model equation, the system of modulation equations is derived up to the second order. At this order of approximation, it is seen that the dispersion relation includes partial derivatives not only of the amplitude but also of the wave vector components. Under some assumptions, a partial differential equation is obtained for the complex amplitude. This equation reveals common features with the modified Korteweg-de Vries equation and with a nonlinear Schrödinger equation. At the first order, it reduces to the cubic Schrödinger equation which had been directly obtained by several authors. Finally, the theory is applied to a plasma wave example.
\end{abstract}

1. Introduction. During the last decade, following the pioneering work of Whitham $[1,2]$, a considerable amount of work has been done on nonlinear dispersive wave modulation, particularly when the wave amplitude is small. In the latter case, when dispersive and nonlinear effects are of comparable amplitudes, various asymptotic methods have been developed for studying the slow nonlinear modulation of a monochromatic plane wave $[3,4]$. Using the so-called "derivative expansion method", as introduced by Sturrock [5], Kawahara [6] showed recently that, within the first order of approximation, the complex amplitude is slowly varying according to a cubic Schrödinger equation. This equation had previously been obtained by Taniuti and Yajima [7] as governing the far field modulation of solutions of a class of nonlinear wave systems (see also Taniuti [8] for other references). Kawahara develops his method by considering the model equation

$$
\left(\frac{\partial^{2}}{\partial t^{2}}-\frac{\partial^{2}}{\partial x^{2}}-\frac{\partial^{4}}{\partial t^{2} \partial x^{2}}\right) f=\frac{\partial^{2}}{\partial x^{2}}\left(\frac{f^{2}}{2}\right) \text {. }
$$

He assumes that the mean value of the non-modulated wave is zero and that the zerothorder approximations of the frequency and wave number are not modulated. Even more recently, Kakutani and Sugimoto [9], henceforth designated as K.S., starting with the same equation and under the same hypotheses, found a similar result by applying an improved version of an extension of the Krylov-Bogoliubov-Mitropolsky method devised by Montgomery and Tidman [3]. Kakutani and Sugimoto apply their results to some problems of plasma wave propagation for which the coefficients of the nonlinear Schrödinger equation are calculated.

* Received February 8, 1977. The author is indebted to Professor T. B. Benjamin for his invitation to the Fluid Mechanics Research Institute of Essex University where the writing of this paper was carried out. 
The purpose of this article is to show how an appropriate two-timing formalism may be applied to an equation or a system of this type, leading to similar results with simpler calculations, and even giving the general system of modulation equations when the restrictive hypotheses are laid aside. The method is deduced from the now-familiar twotiming procedures for fully nonlinear dispersive waves as introduced by Luke [10] (see also Whitham [11]). The formalism used here is rather an appropriate form, for near-linear cases, of a version of such procedures proposed by the author in previous works [12, 13]. It allows the parameters that enter the expansion to be given a physical sense. Moreover, the equations governing the slow variations of the required approximations of these parameters are obtained without superfluous expansions in powers of the scale rate.

At the first stage, one obtains the classical equations of geometrical optics for linear dispersive waves. The following stages provide a system of equations for the first- or second-order approximate values of parameters (frequency, wave number vector, fundamental amplitude and mean value). This system is shown to reduce to the cubic Schrödinger equation for the complex amplitude when one takes the aforesaid restrictive hypotheses into account.

The relative simplicity of the method allows the expansion to be taken to one order further. With similar assumptions to those made in K.S., one obtains in particular the equation for the second order approximation of the complex amplitude. This equation reveals common features with some nonlinear Schrödinger equation and with the so-called modified Korteweg-de Vries equation. It is interesting to note that, in the general case, the dispersion relation is generalized at second and third order by an equation in which appear simultaneously the first and second derivatives not only of the amplitude, as remarked previously by various authors, but also of the frequency and wave number.

In Sec. 2, we present the model equation dealt with, discuss the two-timing asymptotic procedure and perform the substitution up to second-order terms. In Sec. 3, we take the K.S. assumptions into account and show how their particular results may be derived from the preceding theory. Sec. 4 is devoted to an example of ion-acoustic waves, also handled by these authors, in order to show how the general theory may be applied to an actual physical system. Finally, in Sec. 5, the asymptotic procedure is carried on to the next order for the model equation and, under some assumptions, a third-order partial differential equation is derived for the complex amplitude.

2. The two-timing procedure and the results up to second order. We start with the model equation

$$
h^{i j k l} f_{, i j k l}+g^{i j} f_{, i j}=m^{i j}\left(\frac{f^{2}}{2}\right)_{, i j}
$$

for the unknown function $f$ of the independent variables $x^{i}(i=0,1,2,3)$ where $x^{0}=t$ is to be understood as a time variable and the $x^{\alpha}(\alpha=1,2,3)$ as the space variables. Here $f_{, i}$ denotes the partial derivative of $f$ with respect to $x^{i}$ and $h^{i j k l}, g^{i j}$ and $m^{i j}$ are completely symmetric constant tensors (the extension to the case where these tensors are slowly varying functions of the $x^{i}$ is straightforward). The advantage in taking such a generalized form of Kawahara's equation is not so much the fact that it allows three-dimensional propagation, as that it involves indicial notations and the summation convention that are much easier to deal with in displaying the theory.

As we are to study small amplitude solutions, it is convenient to change the unknown function $f$ into $\epsilon \tilde{f}$, where the small parameter $\epsilon$ characterizes that amplitude; so that, dividing by $\epsilon$ and dropping the tilde, the above equation is written as 


$$
h^{i j k l} f_{, i j k l}+g^{i j} f_{, i j}=\epsilon m^{i j}\left(\frac{f^{2}}{2}\right)_{, i j} .
$$

The nonlinear effects appear in the equation in the form of a term of magnitude $\epsilon$ on the right-hand side.

Now we are interested in those approximate solutions for which modulation effects and nonlinear effects are of the same order of magnitude. Then, for the theory we have in view, it is convenient to introduce slow independent variables by

$$
\xi^{i}=\epsilon x^{i} \quad(i=0,1,2,3) .
$$

According to the usual two-timing procedures, as developed by Luke for example, we ought to look for a representation of $f$ of the form:

$$
f=f^{(0)}\left(\varphi, \xi^{i}\right)+\epsilon f^{(1)}\left(\varphi, \xi^{i}\right)+\epsilon^{2} f^{(2)}\left(\varphi, \xi^{i}\right)+\cdots,
$$

where $\varphi$ is a phase variable related to the $x^{i}$ by its slowly varying derivatives

$$
\varphi_{, j}=k_{j}\left(\xi^{i}\right) \quad(j=0,1,2,3) .
$$

However, subsequent considerations will enable us to give the expansion an equivalent form that has meaning from a physical point of view and which renders the further substitution much easier.

The main idea, when substituting the expansion (4) into Eq. (3), is to avoid secular terms in $\varphi$ in order that the expansion be valid at least for a finite range of the slow variables. In doing so, one obtains for $f^{(0)}$ a sinusoidal function of $\varphi$ with non-zero mean value and with period $2 \pi$ when a certain dispersion relation is satisfied by the $k_{\jmath}$. This function $f^{(0)}$ in turn enters the quadratic right-hand side of the linear equation for $f^{(1)}$; so that $f^{(1)}$ appears as the superposition of two non-zero mean valued sinusoidal functions of $\varphi$ with periods $2 \pi$ and $\pi$ respectively. A similar argument may be developed for the higherorder terms: $f^{(p)}$ is then seen to be the superposition of sinusoidal functions of $\varphi$ with periods $2 \pi, \pi, \cdots, 2 \pi /(p+1)$. Now we can group together the terms with the same period (including the mean values as a particular case). If one introduces coefficients that depend on $\epsilon$ and if one changes the phase $\varphi$ for a new phase variable $\theta$ that depends on $\epsilon$ too, one can rewrite the expansion (4), without any loss of generality, in the following form:

$f=A \cos \theta+B+\epsilon\{C \cos (2 \theta)+D \sin (2 \theta)\}+\epsilon^{2}\{E \cos (3 \theta)+F \sin (3 \theta)\}+\cdots$

with

$$
\theta_{, l}=p_{l},
$$

where the parameters $A, B, C, \cdots$ and the $p_{i}$ now depend on $\epsilon$. However, since we are only looking for equations governing the variations of these parameters, we do not need to develop them in $\epsilon$, but rather to keep their approximate values within some appropriate order in these equations. This remark renders the derivation of the modulation equations much more tractable and gives them a more comprehensive form, postponing the possible linearization as a last step. In this form, the formalism is quite similar to the one proposed by the author for the construction of modulated fully nonlinear dispersive wave trains [12]. The physical significance of the parameters is quite clear: A appears to be the real amplitude of the fundamental wave, the phase shift of which has been set equal to zero by redefining the phase $\theta . B$ is the mean value of the wave and the remaining parameters concern the first and following harmonics.

The substitution is now easily performed by equating the corresponding Fourier 
coefficients on both sides of Eq. (3). As mentioned before, the resulting equations will depend on $\epsilon$ and will provide modulation equations governing the evolution of approximations to the parameters. Once the order of the substitution has been chosen, one obtains a finite number of such equations since the order of the coefficients increases with the rank of the harmonics considered.

Before writing down these equations, it is important to notice that, as a first group of equations, valid at any order, we get the consistency equations expressing the fact that the four-covector $p_{t}$ is a gradient by its definition:

$$
p_{i ; j}=p_{j ; i} \quad(i, j=0,1,2,3) .
$$

From now on, we use the notation "; $i$ " for the partial derivative of any function with respect to $\xi^{i}$.

Also we introduce the following functions of the components of the covector $p_{i}$ :

$$
\begin{aligned}
\mathscr{D}\left(p_{s}\right) & =h^{i j k l} p_{i} p_{j} p_{k} p_{l}-g^{i j} p_{i} p_{j}, \\
\mathfrak{T}\left(p_{s}\right) & =-m^{i j} p_{i} p_{j},
\end{aligned}
$$

with the notation $\mathscr{D}^{1 i}$ and $\mathfrak{T}^{1 i}$ for their derivatives with respect to $p_{i}$.

In order to obtain systems of equations connecting the variations of suitably associated approximate values of the various parameters, it is necessary to require different orders of approximation for the different Fourier coefficients. For the purpose of the present section, we need the constant term up to $\epsilon^{3}$, the coefficients of $\cos \theta$ and $\sin \theta$ up to $\epsilon^{2}$ and the coefficients of $\cos 2 \theta$ and $\sin 2 \theta$ up to $\epsilon$. We then obtain respectively, after some eventual divisions by $\epsilon$ or $\epsilon^{2}$ :

$$
\begin{aligned}
& g^{i j} B_{i j i^{1}}=\frac{\epsilon}{2} m^{i j}\left\{\frac{A^{2}}{2}+B^{2}\right\}_{: i j}^{0}, \\
& \{D A\}^{I I}-\frac{\epsilon^{2}}{2}\left\{\left(D^{\mid i j} A_{; i}\right)_{; j}+2 A h^{i j k l}\left(3 p_{k ; i} p_{l ; j}+4 p_{k} p_{l ; i j}\right)\right\}^{0}=\epsilon\{\mathfrak{T H} A B\}^{1}+\frac{\epsilon^{2}}{2}\{\mathfrak{T H} A C\}^{0} \text {, } \\
& \left\{D^{\mid i} A_{; i}+\frac{1}{2} \mathscr{D}_{;} i^{i i} A\right\}^{1}=\frac{\epsilon}{2}\left\{2 \mathscr{M}^{\mid i}(A B)_{; i}+\mathfrak{M}_{; i^{\prime i}} A B+\mathscr{T} A D\right\}^{0}, \\
& \left\{D_{(2)} C\right\}^{0}=\left\{\mathfrak{T T} A A^{2}\right\}^{0}, \\
& \left\{D_{(2)} D\right\}^{0}=0 \text {, }
\end{aligned}
$$

where the 0 or the roman numbers mean that the approximate values of the corresponding order must be understood for the parameters entering the brace so marked. Following Kawahara, we also introduce the notation:

$$
D_{(2)}=\mathscr{D}\left(2 p_{i}\right) .
$$

Dividing by $A$ (the zeroth-order value of which is assumed not to be null) or multiplying by $2 A$ respectively, we may rewrite Eqs. (10) and (11) in the form:

$$
\begin{aligned}
& D^{\mathrm{II}}-\frac{\epsilon^{2}}{2}\left\{\frac{1}{A}\left(D^{\mid i j} A_{; i}\right)_{; j}+2 h^{i j k l}\left(3 p_{k ; i} p_{l ; j}+4 p_{k} p_{l ; i j}\right)\right\}^{0}=\epsilon\{\mathfrak{T} C B\}^{1}+\frac{\epsilon^{2}}{2}\{\mathfrak{I T C} C\}^{0},
\end{aligned}
$$

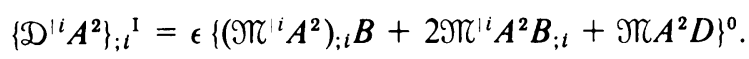

From these equations, we first obtain the classical system of linear geometrical optics 
for the variations of $p_{i}{ }^{\circ}$ and $A^{0}$. As a matter of fact, dropping the 0-symbol, we may write

$$
\begin{gathered}
D\left(p_{i}\right)=0, \\
\left\{D^{\mid l} A^{2}\right\}_{; i}=0,
\end{gathered}
$$

together with the consistency equations (7). Eq. (14) expresses the fact that the slowly varying four-covector $p_{i}$ satisfies the linear dispersion relation. Eq. (15) appears as a waveaction equation as introduced by Hayes [14]. It may also be viewed as a conservation equation for the energy of the fundamental vibration in a tube of "group lines", where the group lines are defined in space-time, with the usual group velocity four-vector $D^{1 i}$, by the proportionality relation

$$
d \xi^{i}:: \mathscr{D}^{1 i} .
$$

It is classical to deduce from Eqs. (7) and (14) that the components $D^{1 i}$ are constant along a group line and consequently that the group lines are straight lines.

At the following stage, we obtain a system linking the slow variations of the approximate values $p_{i}{ }^{1}, A^{1}$ and $B^{0}$. The first two equations, corresponding to (9) and $\left(10^{\prime}\right)$, together with Eqs. (7), govern the coupled variations of $p_{i}{ }^{\mathrm{I}}$ and $B^{0}$. They may be written:

$$
\begin{gathered}
g^{i j} B_{i j j^{0}}=0, \\
D^{I}=\epsilon\left\{\mathfrak{T}(B\}^{0} .\right.
\end{gathered}
$$

Owing to the fact that the linearized model equation is genuinely dispersive, the function $D\left(p_{i}\right)$ is a nonhomogeneous function of the $p_{i}$, so that we certainly have $D_{(2)}{ }^{0} \neq 0$. Then, from Eq. (13), we get:

$$
D^{0}=0,
$$

and Eq. $\left(11^{\prime}\right)$ takes the form

$$
\left\{D^{\mid i} A^{2}\right\}_{; i}{ }^{1}=\epsilon\left\{\left(\mathfrak{T I}^{1 i} A^{2}\right)_{; i} B+2 \mathfrak{M}^{\mid i} A^{2} B_{;}\right\}^{0} .
$$

In the particular case $B^{0}=0$, these equations may be identified with the first-order equations of linear geometrical optics which, a classical result, are identical to the zerothorder ones. So, Eqs. (16) to (18) represent the effect of the nonlinear interaction between the mean value and the fundamental wave on the linear dispersion of this wave. The fourcovector $p_{i}{ }^{1}$ no longer satisfies the linear dispersion relation. There are several possible choices for the first-order group lines. One of them would be simply given by

$$
d \xi^{i}::\left\{D^{1 i}\right\}^{\mathrm{I}} \text {. }
$$

But a better choice seems to be:

$$
d \xi^{i}::\left\{D^{i i}\right\}^{1}-\epsilon\left\{\mathscr{T C}^{\mid i} B\right\}^{0},
$$

where the four-vector appears to be the partial derivative with respect to $p_{i}$ of the modified dispersion function that enters the first-order dispersion equation (17). Hence, it appears as a first-order group velocity. However, whatever choice is retained, the group lines are no longer straight lines, unless $B^{\circ}$ be a constant. Neither is the energy of the fundamental wave conserved in a tube. With the second choice for the $\epsilon$-rays above, it is convenient to write the wave-action equation in the form:

$$
\left\{\left(D^{\mid i}-\epsilon \mathfrak{T}^{\mid i} B\right) A^{2}\right\}_{; i}=\epsilon \mathfrak{T}^{\mid i} B_{;} A^{2},
$$


where the order indices have been omitted. We get a conservation equation along an $\epsilon$-ray tube only in the case where $B^{0}$ is a constant.

The system of Eqs. (9) to (13) allows us to take one step forward by linking the variations of the values $p_{i}{ }^{11}, B^{1}$ and $A^{1}$. To this end, notice that, by the same argument given for $D^{0}$, we can deduce from Eq. (12) the relation

$$
C^{0}=\left\{\frac{\mathscr{M}}{\mathfrak{D}_{(2)}} A^{2}\right\}^{0} .
$$

Then, together with Eqs. (7) and Eq. (18) which remains unchanged, we associate Eq. (9) and the dispersion equation $\left(10^{\prime}\right)$ rewritten as:

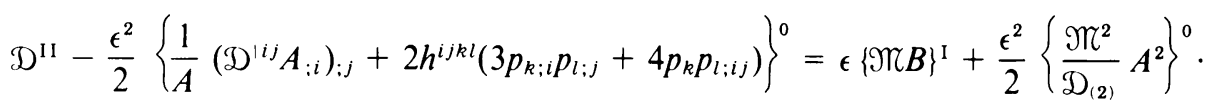

It is noteworthy that the linear part of this dispersion equation, namely the left-hand side, contains derivatives of the parameters up to the second order. The presence of the second-order derivatives of the amplitude has already been pointed out by different authors dealing with near-linear or even fully non-linear (see [12] for example) dispersive waves and has recently been suggested as a general feature by Whitham in his treatise [15]. However, the appearance at this stage of the derivatives of frequency and wave number vector seems not to have been often encountered. Their appearance necessitates dealing with at least fourth-order partial differential equations and carrying out the asymptotic procedure to second order, with non-constant zeroth-order value of the frequency and wave number.

At this stage, we would be able to derive the wave-action equation for the variations of $A^{11}$. However, we would need the value of $D^{1}$ and we postpone the writing of this equation to Sec. 5, where we derive a system for $p_{i}{ }^{\mathrm{II}}, A^{\mathrm{II}}$ and $B^{\mathrm{II}}$.

3. How the cubic Schrödinger equation arises. In the expression of the covector $p_{i}$, it is open to us to distinguish the zeroth-order part by writing

$$
p_{i}=q_{i}+\epsilon \tilde{p}_{i},
$$

where $q_{i}$ must be the gradient of some phase function $\psi\left(x^{k}\right)$ and $\tilde{p}_{i}$ may be considered as the gradient of some function $\alpha$ of $\xi^{k}$ and $\epsilon$. This amounts to saying that the $\epsilon$-dependent phase $\theta$ is related to the $\epsilon$-independent one $\psi$ by the relation

$$
\theta=\psi+\alpha\left(\xi^{k}, \epsilon\right) \text {. }
$$

We may still write the relation between $p_{i}$ and $q_{i}$ as:

$$
p_{i}=q_{i}+\epsilon \alpha_{; i} .
$$

Now, on account of the K.S. assumptions, we are to study the modulations of a plane harmonic wave that extends in the whole space-time when the nonlinear effects of the interacting mean value are small. Accordingly, we assume that the $q_{i}$ are constant with regard to the $\xi$ variables and that the mean value is of order $\epsilon$. In this case, the $\epsilon$ independent phase $\psi$ is merely the common linear form $\psi=q_{i} x^{i}$ and the zeroth-order rays are parallel straight lines.

Let us put

$$
B=\epsilon \tilde{B},
$$


and rewrite the system of Eqs. (9), (18) and (21) (Eqs. (7) are no longer useful since we have introduced the function $\alpha$ ):

$$
\begin{gathered}
g^{i j} \tilde{B}_{; i j}{ }^{0}=\frac{1}{4} m^{i j}\left(A^{2}\right)_{; i j}{ }^{0}, \\
\left\{D^{\mid i} A^{2}\right\}_{; i}{ }^{\mathrm{I}}=0, \\
\{D A\}^{\mathrm{II}}-\frac{\epsilon^{2}}{2}\left\{D^{\mid i j} A_{; i j}\right\}^{0}=\epsilon^{2}\left\{\mathfrak{T}\left(\tilde{B}+\frac{\mathfrak{T}}{2 D_{(2)}} A^{2}\right) A\right\}^{0} .
\end{gathered}
$$

Here the argument in $D^{I I}$ or $D^{I}$ and its derivatives is $p_{i}$ as given by (22). So it is convenient to expand these functions in Taylor series. At first sight, it would seem that we need the value $A^{\text {II }}$ in (26). However, owing to the dispersion relation (14), we have

$$
D^{0}=D\left(q_{i}\right)=0,
$$

so that only $A^{1}$ enters Eq. (26). When the Taylor expansion is performed, Eqs. (25) and (26) take the forms

$$
\begin{aligned}
& \mathscr{D}^{1 i} A_{; i}{ }^{\mathrm{I}}+\frac{\epsilon}{2} \mathscr{D}^{1 i j}\left\{2 A_{; i} \alpha_{; j}+A^{2} \alpha_{; i j}\right\}^{0}=0, \\
& \mathscr{D}^{\mid i}\left\{A \alpha_{; i}\right\}^{1}+\frac{\epsilon}{2} \mathscr{D}^{\mid i j}\left\{A \alpha_{; i} \alpha_{; j}-A_{; i j}\right\}^{0}=\epsilon\left\{\mathfrak{M}\left(\tilde{B}+\frac{\mathscr{M}}{2 D_{(2)}} A^{2}\right) A\right\}^{0},
\end{aligned}
$$

where the $D^{1 i}$ and $D^{1 i j}$ are now constant as functions of the $q_{i}$ only.

Forming the combination $\{(27)+\mathrm{i}(28)\} \exp (\mathrm{i} \alpha)$, where $\mathrm{i}$ is the usual complex squareroot of -1 , and introducing the complex amplitude $a$ by

$$
a=A \exp (\mathrm{i} \alpha)
$$

we obtain the following equation:

$$
\mathbb{D}^{\mid i} a_{; i}{ }^{1}-\mathrm{i} \frac{\epsilon}{2} \mathscr{D}^{i i j} a_{; i j}{ }^{0}=\mathrm{i} \epsilon \mathfrak{T}\left\{\left(\tilde{B}+\frac{\mathfrak{M}}{2 D_{(2)}} a \bar{a}\right) a\right\}^{0},
$$

where $\bar{a}$ denotes the complex conjugate value of $a$. The first derivative term in the left-hand side of (29) represents differentiation along the zeroth-order rays. For $a^{0}$, the equation would merely read

$$
\mathscr{D}^{i i} a_{; i}^{0}=0,
$$

which expresses that zeroth-order values of amplitude and phase shift are constant along the (parallel) rays.

As $\tilde{B}$ appears in Eq. (29), this equation must be associated with Eq. (24) rewritten as

$$
g^{i j} \tilde{B}_{; i j}{ }^{0}=\frac{1}{4} m^{i j}(a \bar{a})_{; i j}{ }^{0},
$$

and (29), (31) is the right system to be considered for $a^{\mathrm{I}}$ and $\tilde{B}^{0}$ in this problem. However, if in addition, following Kakutani and Sugimoto, we assume that $\tilde{B}$ is a function of $a$ and $\bar{a}$, hence a constant along the rays, and that the space-time is two-dimensional, then (31) turns out to be an ordinary differential equation. By integrating this equation with an extra non-secular condition relative to the $\xi$ variables, we simply obtain

$$
\tilde{B}=b a \bar{a}+\beta,
$$


where $b$ is a known function of $q_{i}$ and $\beta$ an integration constant which could be determined by some initial condition. Substitution of (32) into (29) leads then to the so-called cubic Schrödinger equation:

$$
D^{i i} a_{; i}{ }^{\mathrm{I}}-\mathrm{i} \frac{\epsilon}{2} \mathscr{D}^{1 i j} a_{; i j}{ }^{0}=\mathrm{i} \epsilon \mathfrak{M}\left\{\left[\left(b+\frac{\mathscr{N}}{2 D_{(2)}}\right) a \dot{a}+\beta\right] a\right\}^{0},
$$

which is the result referred to in the introduction.

4. A plasma wave example. As an example of application of the theory derived in Sec. 2 to a physical system, let us consider the propagation of the ion-acoustic waves in a plasma. This example has been studied by K.S. in the scope of their K.B.M. theory and the reader is referred to their article for the derivation of the physical system. The relevant non-dimensional system is:

$$
\frac{\partial n}{\partial t}+\frac{\partial}{\partial x}(n u)=0, \quad \frac{\partial n_{e}}{\partial x}=-n_{e} E, \quad \frac{\partial u}{\partial t}+u \frac{\partial u}{\partial x}=E, \quad \frac{\partial E}{\partial x}=n-n_{e},
$$

where $n, n_{e}, u$ and $E$ denote, respectively, ion density, electron density, ion-fluid velocity and the electric field. (The system has been derived under the following hypotheses: null ion temperature, constant $(\neq 0)$ electron temperature; the electron inertia is neglected and the plasma is collision-free.) The first three equations express conservation of number of ions, electron momentum and ion momentum. The last one is the Poisson equation for the electric field.

As is easily seen, the system (34) admits a particular solution corresponding to the equilibrium rest state of the gas:

$$
n=n_{e}(=1, \text { say }), \quad u=0, \quad E=0 .
$$

We now study the small-amplitude perturbations around that equilibrium state by setting:

$$
n=1+\epsilon \tilde{n}, \quad n_{e}=1+\epsilon \tilde{n}_{e}, \quad u=\epsilon \tilde{u}, \quad E=\epsilon \tilde{E} .
$$

We then obtain the following system, after dividing once by $\epsilon$ :

$$
\begin{gathered}
\frac{\partial \tilde{n}}{\partial t}+\frac{\partial \tilde{u}}{\partial x}=-\epsilon \frac{\partial}{\partial x}(\tilde{n} \tilde{u}), \quad \frac{\partial \tilde{n}_{e}}{\partial x}+\tilde{E}=-\epsilon \tilde{n_{e}} \tilde{E}, \\
\frac{\partial \tilde{u}}{\partial t}-\tilde{E}=-\epsilon \tilde{u} \frac{\partial \tilde{u}}{\partial x}, \quad \frac{\partial \tilde{E}}{\partial x}-\tilde{n}+\tilde{n}_{e}=0 .
\end{gathered}
$$

This corresponds to Eq. (3), where the nonlinear terms have been written in the right-hand side.

The linearized system (35), obtained by putting $\epsilon=0$, admits the exact harmonic solution

$$
\mid \begin{aligned}
\tilde{n} & =N_{0}+K \omega\left(k^{2}+1\right) \sin \theta \\
\tilde{n}_{e} & =N_{0}+K \omega \sin \theta \\
\tilde{u} & =U_{0}+K k \sin \theta \\
\tilde{E} & =K k \omega \cos \theta,
\end{aligned}
$$

with $\theta=\omega t-k x$, provided that $\omega, k$ satisfy the dispersion relation: 


$$
D(\omega, k) \equiv \omega^{2} k^{2}+\omega^{2}-k^{2}=0 .
$$

The densities $\tilde{n}, \tilde{n}_{e}$ and the velocity $\tilde{u}$ are seen to have the same phase. whereas the electric field $\tilde{E}$ has a $\pi / 2$ phase shift.

We now study slowly modulated trains of waves of the preceding form, taking dispersive and nonlinear effects into account. Accordingly, as a generalization of (5), we look for the unknowns in the asymptotic form

$$
\mid \begin{aligned}
\tilde{n} & =N_{0}+N_{1} \cos \theta+N_{1}{ }^{\prime} \sin \theta+\epsilon\left(N_{2} \cos (2 \theta)+N_{2}{ }^{\prime} \sin (2 \theta)\right)+O\left(\epsilon^{2}\right) \\
\tilde{n}_{e} & =M_{0}+M_{1} \cos \theta+M_{1}{ }^{\prime} \sin \theta+\epsilon\left(M_{2} \cos (2 \theta)+M_{2}{ }^{\prime} \sin (2 \theta)\right)+O\left(\epsilon^{2}\right) \\
\tilde{u} & =U_{0}+U_{1} \cos \theta+U_{1}^{\prime} \sin \theta+\epsilon\left(U_{2} \cos (2 \theta)+U_{2}{ }^{\prime} \sin (2 \theta)\right)+O\left(\epsilon^{2}\right) \\
\tilde{E} & =E_{0}+E_{1} \cos \theta+E_{1}{ }^{\prime} \sin \theta+\epsilon\left(E_{2} \cos (2 \theta)+E_{2}{ }^{\prime} \sin (2 \theta)\right)+O\left(\epsilon^{2}\right),
\end{aligned}
$$

where $\theta$ is the phase variable related to $x, t$ by its derivatives

$$
\partial \theta / \partial t=\omega, \quad \partial \theta / \partial x=-k
$$

and where $\omega, k, N_{0}, N_{1}$, etc. are functions of the parameter $\epsilon$ and of the slow independent variables

$$
\tau=\epsilon t, \quad \xi=\epsilon x .
$$

Owing to the particular role played by $\tilde{E}$ in the exact solution (36), we choose $\theta$ in such a way that, at any order,

$$
E_{1}{ }^{\prime}=0
$$

As a consistency equation, we have now

$$
(\partial \omega / \partial \xi)+(\partial k / \partial \tau)=0 .
$$

Substituting the expansions (38) into the system (35) and identifying the various Fourier coefficients on both sides of each equation, to order $\epsilon^{2}$, and omitting here the order indices, we obtain the following equations.

i) For the $\theta$-independent terms:

$$
\begin{gathered}
\epsilon\left(\frac{\partial N_{0}}{\partial \tau}+\frac{\partial U_{0}}{\partial \xi}\right)=-\epsilon^{2} \frac{\partial}{\partial \xi}\left(N_{0} U_{0}+\frac{1}{2} N_{1} U_{1}+\frac{1}{2} N_{1}{ }^{\prime} U_{1}{ }^{\prime}\right) \\
E_{0}+\epsilon \frac{\partial M_{0}}{\partial \xi}=-\epsilon\left(M_{0} E_{0}+\frac{1}{2} M_{1} E_{1}\right), \\
E_{0}-\epsilon \frac{\partial U_{0}}{\partial \tau}=\frac{\epsilon^{2}}{2} \frac{\partial}{\partial \xi}\left(U_{0}{ }^{2}+\frac{1}{2} U_{1}{ }^{2}+\frac{1}{2} U_{1}{ }^{2}\right), \\
-N_{0}+M_{0}+\epsilon \frac{\partial E_{0}}{\partial \xi}=0 .
\end{gathered}
$$

ii) The $\cos \theta$ and $\sin \theta$ coefficient systems are considered simultaneously. In fact, at each order, the whole system turns out to split into two independent systems: one for the unknowns $\left(N_{1}, M_{1}, U_{1}\right)$ :

$$
\omega N_{1}-k U_{1}-\epsilon\left(\frac{\partial N_{1}{ }^{\prime}}{\partial \tau}+\frac{\partial U_{1}{ }^{\prime}}{\partial \xi}\right)=\epsilon k\left(N_{0} U_{1}+U_{0} N_{1}\right)+\epsilon^{2} \frac{\partial}{\partial \xi}\left(N_{0} U_{1}{ }^{\prime}+U_{0} N_{1}{ }^{\prime}\right)
$$




$$
\begin{gathered}
+\frac{\epsilon^{2}}{2} k\left(N_{1} U_{2}+U_{1} N_{2}+N_{1}^{\prime} U_{2}^{\prime}+U_{1}^{\prime} N_{2}^{\prime}\right), \\
k M_{1}+\epsilon \frac{\partial M_{1}^{\prime}}{\partial \xi}=-\epsilon E_{0} M_{1}^{\prime}-\frac{\epsilon^{2}}{2}\left(M_{1} E_{2}^{\prime}+E_{1} M_{2}^{\prime}-M_{1}^{\prime} E_{2}\right), \\
\omega U_{1}-\epsilon \frac{\partial U_{1}^{\prime}}{\partial \tau}=\epsilon k U_{0} U_{1}+\epsilon^{2} \frac{\partial}{\partial \xi}\left(U_{0} U_{1}^{\prime}\right)+\frac{\epsilon^{2}}{2} k\left(U_{1} U_{2}+U_{1}^{\prime} U_{2}^{\prime}\right), \\
-N_{1}+M_{1}+\epsilon\left(\partial E_{1} / \partial \xi\right)=0
\end{gathered}
$$

and the second one for $\left(N_{1}{ }^{\prime}, M_{1}{ }^{\prime}, U_{1}{ }^{\prime}, E_{1}\right)$ :

$$
\begin{aligned}
& \omega N_{1}{ }^{\prime}-k U_{1}^{\prime}+\epsilon\left(\frac{\partial N_{1}}{\partial \tau}+\frac{\partial U_{1}}{\partial \xi}\right)=\epsilon k\left(N_{0} U_{1}{ }^{\prime}+U_{0} N_{1}{ }^{\prime}\right)-\epsilon^{2} \frac{\partial}{\partial \xi}\left(N_{0} U_{1}+U_{0} N_{1}\right) \\
& +\frac{\epsilon^{2}}{2} k\left(N_{1} U_{2}^{\prime}+U_{1} N_{2}^{\prime}-N_{1}^{\prime} U_{2}-U_{1}{ }^{\prime} N_{2}\right), \\
& k M_{1}^{\prime}-E_{1}-\epsilon \frac{\partial M_{1}}{\partial \xi}=\epsilon\left(M_{0} E_{1}+E_{0} M_{1}\right)+\frac{\epsilon^{2}}{2}\left(M_{1} E_{2}+E_{1} M_{2}+M_{1}^{\prime} E_{2}^{\prime}\right), \\
& \omega U_{1}^{\prime}-E_{1}+\epsilon \frac{\partial U_{1}}{\partial \tau}=\epsilon k U_{0} U_{1}^{\prime}-\epsilon^{2} \frac{\partial}{\partial \xi}\left(U_{0} U_{1}\right)+\frac{\epsilon^{2}}{2} k\left(U_{1} U_{2}^{\prime}-U_{1}^{\prime} U_{2}\right), \\
& k E_{1}-N_{1}^{\prime}+M_{1}^{\prime}=0 .
\end{aligned}
$$

The system (42), with four equations for only three unknowns, leads to a compatibility relation which will appear to be the relevant wave-action equation. The system (43) which must be singular at zeroth order will provide the dispersion equation at various orders.

iii) In a similar way, we associate the $\cos 2 \theta$ and $\sin 2 \theta$ coefficient systems, only needed here at the lowest order, so that we obtain a system for the unknowns $\left(N_{2}, M_{2}, U_{2}, E_{2}{ }^{\prime}\right)$ :

$$
\begin{array}{cc}
2 \omega N_{2}-2 k U_{2}=k\left(N_{1} U_{1}-N_{1}{ }^{\prime} U_{1}{ }^{\prime}\right), & 2 k M_{2}+E_{2}{ }^{\prime}=-\frac{1}{2} M_{1}{ }^{\prime} E_{1}, \\
2 \omega U_{2}+E_{2}{ }^{\prime}=\frac{k}{2}\left(U_{1}^{2}-U_{1}^{\prime 2}\right), & 2 k E_{2}{ }^{\prime}+N_{2}-M_{2}=0,
\end{array}
$$

and another system for $\left(N_{2}{ }^{\prime}, M_{2}{ }^{\prime}, U_{2}{ }^{\prime}, E_{2}\right)$ :

$$
\begin{gathered}
2 \omega N_{2}{ }^{\prime}-2 k U_{2}^{\prime}=k\left(N_{1} U_{1}^{\prime}+U_{1} N_{1}^{\prime}\right), \quad 2 k M_{2}^{\prime}-E_{2}=\frac{1}{2} M_{1} E_{1}, \\
2 \omega U_{2}^{\prime}-E_{2}=k U_{1} U_{1}^{\prime}, \quad 2 k E_{2}-N_{2}{ }^{\prime}+M_{2}^{\prime}=0 .
\end{gathered}
$$

The system (41) at the lowest order gives the following relations for the zeroth-order mean values:

$$
\begin{aligned}
& E_{0}{ }^{0}=0, \quad M_{0}{ }^{0}=N_{0}{ }^{0}, \\
& \frac{\partial N_{0}^{0}}{\partial \tau}+\frac{\partial U_{0}^{0}}{\partial \xi}=0 .
\end{aligned}
$$

At zeroth order, we deduce from (42):

$$
N_{1}{ }^{0}=M_{1}{ }^{0}=U_{1}{ }^{0}=0,
$$

without any further condition, while system (43) takes the form of the following homogeneous algebraic system: 


$$
\begin{aligned}
\left\{\omega N_{1}{ }^{\prime}-k U_{1}{ }^{\prime}\right\}^{0} & =0, \\
\left\{k M_{1}^{\prime}-E_{1}\right\}^{0} & =0, \\
\left\{\omega U_{1}^{\prime}-E_{1}\right\}^{0} & =0, \\
\left\{k E_{1}-N_{1}{ }^{\prime}+M_{1}^{\prime}\right\}^{0} & =0 .
\end{aligned}
$$

The solution of (49) may be expressed as

$$
N_{1}{ }^{0}=\left\{\frac{k}{\omega^{2}} E_{1}\right\}^{0}, \quad M_{1}{ }^{0}=\left\{\frac{1}{k} E_{1}\right\}^{0}, \quad U_{1}{ }^{0}=\left\{\frac{1}{\omega} E_{1}\right\}^{0},
$$

provided that the determinant of the system is zero. This requirement leads to the dispersion relation (37) to be satisfied by $\omega^{0}, k^{0}$.

Returning to system (41) at first order, we deduce the equation

$$
\frac{\partial N_{0}^{0}}{\partial \xi}+\frac{\partial U_{0}^{0}}{\partial \tau}=0
$$

as a compatibility condition. With Eq. (47), this shows that $N_{0}{ }^{0}$ and $U_{0}{ }^{0}$ satisfy the wave equation

$$
\frac{\partial^{2} N_{0}^{0}}{\partial \tau^{2}}-\frac{\partial^{2} N_{0}^{0}}{\partial \xi^{2}}=0
$$

which is similar to Eq. (16) of Sec. 2. Then we obtain for the first-order mean values:

$$
\begin{aligned}
& E_{0}{ }^{\mathrm{I}}=-\epsilon \frac{\partial N_{0}{ }^{0}}{\partial \xi}=\epsilon \frac{\partial U_{0}^{0}}{\partial \tau}, \\
& M_{0}{ }^{\mathrm{I}}=N_{0}^{\mathrm{I}},
\end{aligned}
$$

together with an equation binding $N_{0}{ }^{\mathrm{I}}$ and $U_{0}{ }^{\mathrm{I}}$ :

$$
\frac{\partial N_{0}^{\mathrm{I}}}{\partial \tau}+\frac{\partial U_{0}^{\mathrm{I}}}{\partial \xi}=-\epsilon \frac{\partial}{\partial \xi}\left\{N_{0} U_{0}+\frac{k}{2 \omega^{3}} E_{1}{ }^{2}\right\}^{0} \text {. }
$$

At the same order, the system (42) gives the values:

$$
\mid \begin{aligned}
& N_{1}{ }^{\mathrm{I}}=\epsilon\left\{\frac{\partial E_{1}}{\partial \xi}-\frac{1}{k} \frac{\partial}{\partial \xi}\left(\frac{E_{1}}{k}\right)\right\}^{0}, \\
& M_{1}{ }^{\mathrm{I}}=-\epsilon\left\{\frac{1}{k} \frac{\partial}{\partial \xi}\left(\frac{E_{1}}{k}\right)\right\}^{0}, \\
& U_{1}{ }^{\mathrm{I}}=\epsilon\left\{\frac{1}{\omega} \frac{\partial}{\partial \tau}\left(\frac{E_{1}}{\omega}\right)\right\}^{0},
\end{aligned}
$$

together with a compatibility condition which may be written:

$$
\left\{\frac{k}{\omega} \frac{\partial}{\partial \tau}\left(\frac{E_{1}}{\omega}\right)+\frac{\partial}{\partial \tau}\left(\frac{k}{\omega^{2}} E_{1}\right)+\frac{\omega}{k} \frac{\partial}{\partial \xi}\left(\frac{E_{1}}{k}\right)+\frac{\partial}{\partial \xi}\left(\frac{E_{1}}{\omega}\right)-\omega \frac{\partial E_{1}}{\partial \xi}\right\}^{0}=0 .
$$

Multiplying the latter equation by $E_{1} / \omega$ and using the dispersion relation (37) for $\omega^{0}$ and $k^{0}$, one rewrites this equation in the conservative form

$$
\frac{\partial}{\partial \tau}\left\{\frac{k}{\omega^{3}} E_{1}{ }^{2}\right\}^{0}+\frac{\partial}{\partial \xi}\left\{\frac{1}{k^{2}} E_{1}{ }^{2}\right\}^{0}=0
$$


or equivalently, by using the consistency equation (40), in the form

$$
\frac{\partial}{\partial \tau}\left\{D_{\omega} E_{1}{ }^{2}\right\}^{0}-\frac{\partial}{\partial \xi}\left\{D_{k} E_{1}{ }^{2}\right\}^{0}=0,
$$

which is Eq. (15) of Sec. 2.

Finally, the system (43) may now be written

$$
\begin{aligned}
& \left\{\omega N_{1}^{\prime}-k U_{1}^{\prime}\right\}^{1}=\epsilon\left\{k\left(\frac{N_{0}}{\omega}+\frac{\bar{k}}{\omega^{2}} U_{0}\right) E_{1}\right\}^{0}, \\
& \left\{k M_{1}^{\prime}-E_{1}\right\}^{1}=\epsilon\left\{N_{0} E_{1}\right\}^{0}, \\
& \left\{\omega U_{1}^{\prime}-E_{1}\right\}^{1}=\epsilon\left\{\frac{k}{\omega} U_{0} E_{1}\right\}^{0}, \\
& \left\{k E_{1}-N_{1}^{\prime}+M_{1}^{\prime}\right\}^{1}=0,
\end{aligned}
$$

and, by eliminating $N_{1}{ }^{\prime 1}, M_{1}{ }^{1}, U_{1}{ }^{1}$, owing to the fact that the determinant of the left-hand side of the system is $D^{1}$, and hence of order $\epsilon$, we are led to a relation which turns out to be homogeneous in $E_{1}{ }^{0}$. A fter the relevant simplification, since $E_{1}{ }^{0}$ is to be nonzero, we obtain the first-order dispersion relation

$$
D^{I}=\epsilon\left\{\omega^{2} k^{2} N_{0}+2 \frac{k^{3}}{\omega} U_{0}\right\}^{0},
$$

which is Eq. (17) of Sec. 2. The system may then be solved to give the values

$$
\left\{\begin{array}{l}
N_{1}{ }^{1}=\left\{\frac{k}{\omega^{2}} E_{1}\right\}^{\mathrm{I}}+\epsilon\left\{\left(\frac{k}{\omega^{2}} N_{0}+2 \frac{k^{2}}{\omega^{3}} U_{0}\right) E_{1}\right\}^{0}, \\
M_{1}{ }^{11}=\left\{\frac{1}{k} E_{1}\right\}^{\mathrm{I}}+\epsilon\left\{\frac{1}{k} N_{0} E_{1}\right\}^{0}, \\
U_{1}{ }^{1}=\left\{\frac{1}{\omega} E_{1}\right\}^{\mathrm{I}}+\epsilon\left\{\frac{k}{\omega^{2}} U_{0} E_{1}\right\}^{0} .
\end{array}\right.
$$

Proceeding now to the second-order equations, we first obtain from system (41) a second equation for $N_{0}{ }^{1}, U_{0}$ :

$$
\frac{\partial N_{0}{ }^{1}}{\partial \xi}+\frac{\partial U_{0}{ }^{1}}{\partial \tau}=-\frac{\epsilon}{2} \frac{\partial}{\partial \xi}\left\{U_{0}{ }^{2}-N_{0}{ }^{2}+\frac{1}{2} E_{1}{ }^{2}\right\}^{0} .
$$

Together with Eq. (54), it corresponds to Eq. (9) of Sec. 2.

Before handling systems (42) and (43), we need the zeroth-order values of the harmonic parameters $N_{2}, N_{2}{ }^{\prime}, \cdots$. These are given by solving systems (44) and (45). The last one leads to the null values

$$
N_{2}{ }^{\circ}=M_{2}{ }^{\prime 0}=U_{2}{ }^{\prime 0}=E_{2}{ }^{0}=0,
$$

since it turns out to be a homogeneous system whose coefficient matrix has non-zero determinant

$$
D_{(2)}{ }^{0} \equiv \mathscr{D}\left(2 \omega^{0}, 2 k^{0}\right) \neq 0 .
$$

The system (44), however, is nonhomogeneous with the same non-zero determinant $\mathfrak{D}_{(2)}{ }^{0}$ and will provide the solution: 


$$
\left\{\begin{array}{l}
N_{2}{ }^{0}=-\left\{\frac{12 k^{4}+15 k^{2}+2}{12 \omega^{2} k^{2}} E_{1}{ }^{2}\right\}^{0}, \\
M_{2}{ }^{0}=-\left\{\frac{4 \omega^{2}+3 k^{2}+2}{12 \omega^{2} k^{2}} E_{1}{ }^{2}\right\}^{0}, \\
U_{2}{ }^{0}=-\left\{\frac{\omega}{k} \frac{6 k^{4}+9 k^{2}+2}{12 \omega^{2} k^{2}} E_{1}{ }^{2}\right\}^{0}, \\
E_{2}{ }^{0}=\left\{\frac{3 k^{4}-\omega^{2}+3 k^{2}}{6 \omega^{2} k^{3}} E_{1}{ }^{2}\right\}^{0} .
\end{array}\right.
$$

From the system (42) at the second order, we then obtain the compatibility condition which may be written, by using the first-order dispersion relation (58), in the form

$$
\begin{aligned}
& \left\{\frac{\partial}{\partial \tau}\left(\frac{k}{\omega^{3}} E_{1}^{2}\right)+\frac{\partial}{\partial \xi}\left(\frac{1}{k^{2}} E_{1}^{2}\right)\right\}^{1} \\
& \quad+\epsilon\left\{\frac{\partial}{\partial \tau}\left(\frac{3 k^{2}}{\omega^{4}} U_{0} E_{1}^{2}\right)+\frac{\partial}{\partial \xi}\left(\frac{k}{\omega^{3}} U_{0} E_{1}^{2}\right)+\left(\frac{1}{2}-\frac{1}{\omega^{2}}\right) E_{1}{ }^{2} \frac{\partial U_{0}}{\partial \tau}\right\}^{0}=0 .
\end{aligned}
$$

In the same way as we treated Syst. (43) at first order, we now obtain at the second order the dispersion relation

$$
\begin{aligned}
D^{\mathrm{II}} & +\epsilon^{2}\left\{\frac{k^{2}}{E_{1}} \frac{\partial}{\partial \tau}\left[\frac{1}{\omega} \frac{\partial}{\partial \tau}\left(\frac{E_{1}}{\omega}\right)\right]+\frac{k \omega}{E_{1}} \llbracket \frac{\partial^{2} E_{1}}{\partial \tau}+\frac{\partial}{\partial \xi}\left[\frac{1}{\omega} \frac{\partial}{\partial \tau}\left(\frac{E_{1}}{\omega}\right)\right]-\frac{\partial}{\partial \tau}\left[\frac{1}{k} \frac{\partial}{\partial \xi}\left(\frac{E_{1}}{k}\right)\right] \rrbracket\right. \\
& \left.-\frac{\omega^{2}}{E_{1}} \frac{\partial}{\partial \xi}\left[\frac{1}{k} \frac{\partial}{\partial \xi}\left(\frac{E_{1}}{k}\right)\right]\right\}^{0}=\epsilon\left\{\left(k^{2}-\omega^{2}\right) N_{0}+\frac{2 k^{3}}{\omega} U_{0}\right\}^{1}+\epsilon^{2}\left\{\frac{k^{3}}{\omega} U_{0}\left(2 N_{0}+\frac{3 k}{\omega} U_{0}\right)\right. \\
& \left.+\frac{24 k^{8}+81 k^{6}+93 k^{4}+42 k^{2}+4}{24 k^{2}\left(k^{2}+1\right)} E_{1}{ }^{2}\right\}^{0}
\end{aligned}
$$

which must be compared with Eq. (21) and for which the same remarks are valid as regards the presence of the second derivatives of the parameters $E_{1}, \omega$ and $k$. We have thus obtained the system of equations (40), (54), (60), (63) and (64) for the coupled slow variations of the approximate values $\omega^{\mathrm{II}}, k^{\mathrm{II}}, N_{0}{ }^{\mathrm{I}}, U_{0}{ }^{1}$ and $E_{1}{ }^{\mathrm{I}}$.

Now, if we take the K.S. assumptions into account, these equations may be simplified by taking

$$
\begin{aligned}
& N_{0}^{0}=U_{0}^{0}=0 \\
& \omega=\mid \omega^{0}+\epsilon \alpha_{\tau} \\
& k=\mid k^{0}-\epsilon \alpha_{\xi}
\end{aligned} \mid
$$

with $\omega^{0}, k^{0}$ constant and $\alpha$ a function of $(\tau, \xi, \epsilon)$. Setting,

$$
N_{0}{ }^{\mathrm{I}}=\epsilon \tilde{N}_{0}, \quad U_{0}^{\mathrm{I}}=\epsilon \tilde{U}_{0}
$$

we rewrite Eqs. (54) and (60) in the form

$$
\begin{aligned}
& \frac{\partial \tilde{N}_{0}}{\partial \tau}+\frac{\partial \tilde{U}_{0}}{\partial \xi}=-\left\{\frac{k}{2 \omega^{3}} \frac{\partial\left(E_{1}^{2}\right)}{\partial \xi}\right\}^{0}, \\
& \frac{\partial \tilde{N}_{0}}{\partial \xi}+\frac{\partial \tilde{U}_{0}}{\partial \tau}=-\frac{1}{4}\left\{\frac{\partial\left(E_{1}^{2}\right)}{\partial \xi}\right\}^{0}
\end{aligned}
$$


while the first-order dispersion equation reduces to

$$
D^{I}=0 .
$$

This last result allows one to write the wave-action equation (63) in the form

$$
\left\{\frac{\partial}{\partial \tau}\left(D_{\omega} E_{1}^{2}\right)-\frac{\partial}{\partial \xi}\left(D_{k} E_{1}^{2}\right)\right\}^{1}=0,
$$

and the second-order dispersion relation as

$$
\begin{aligned}
\left\{D E_{1}\right\}^{11}+ & \epsilon^{2}\left\{\frac{k^{2} \partial^{2} E_{1}}{\omega^{2} \partial \tau^{2}}+2 \omega k \frac{\partial^{2} E_{1}}{\partial \tau \partial \xi}-\frac{\omega^{2}}{k^{2}} \frac{\partial^{2} E_{1}}{\partial \xi^{2}}\right\}^{0} \\
& =\epsilon^{2}\left\{\left(\omega^{2} k^{2} \tilde{N}_{0}+\frac{2 k^{3}}{\omega} \tilde{U}_{0}\right) E_{1}+\frac{24 k^{8}+81 k^{6}+93 k^{4}+42 k^{2}+4}{24 k^{2}\left(k^{2}+1\right)} E_{1}\right\}^{3} .
\end{aligned}
$$

To conform with K.S. notations, it is convenient here to take

$$
a=\frac{1}{2} E_{1} \exp (\mathrm{i} \alpha)
$$

for the complex amplitude of the electric field. Assuming now that $\tilde{N}_{0}$ and $\tilde{U}_{0}$ depend on $\xi$ and $r$ through $a$ and $\dot{a}$, we solve the system (67) to obtain

$$
\begin{aligned}
& \tilde{N}_{0}=\left\{\frac{k^{4}\left(k^{2}+2\right)}{\omega^{6}-k^{6}} a \bar{a}\right\}^{0}+\text { constant, } \\
& \tilde{U}_{0}=\left\{\frac{k^{3}\left(2 k^{4}+\omega^{6}\right)}{\omega^{3}\left(\omega^{6}-k^{6}\right)} a \bar{a}\right\}^{0}+\text { constant. }
\end{aligned}
$$

It may be worth noticing here that we need no extra non-secularity condition relatively to $\tau, \xi$ since we integrate a first-order system of differential equations.

Finally, substituting (70) into (69) and using the same process as in Sec. 3, we obtain the following cubic Schrödinger equation for $a$ :

$\mathscr{D}_{\omega}{ }^{0} \frac{\partial a^{1}}{\partial \tau}-\mathscr{D}_{k}{ }^{0} \frac{\partial a^{1}}{\partial \xi}-\mathbf{i} \frac{\epsilon}{2}\left\{D_{\omega \omega} \frac{\partial^{2} a}{\partial \tau^{2}}-2 D_{\omega k} \frac{\partial^{2} a}{\partial \tau \partial \xi}+D_{k k} \frac{\partial^{2} a}{\partial \xi^{2}}\right\}^{0}=\mathbf{i} \epsilon\{(\lambda a \bar{a}+\mu) a\}^{0}$,

where $\mu$ is some integration constant to be determined by initial conditions when integrating the system (67), and $\lambda$ is the following function of $k^{0}$ :

$$
\lambda=\frac{3 k^{10}+6 k^{8}-6 k^{6}-29 k^{4}-30 k^{2}-12}{6 k^{2}\left(k^{2}+1\right)\left(k^{4}+3 k^{2}+3\right)}
$$

These last results may be seen to be in full accordance with those previously obtained by Kakutani and Sugimoto.

5. Higher-order modulation. There is no difficulty in carrying the asymptotic procedure further. We are now interested in deriving the system for the slow variations of the parameters at the next order and, with the relevant assumptions, in finding the stepped forward analogue of the system (29), (31) of Sec. 3. As a particular case, we would obtain the equation that generalizes (33) for the second-order value of the complex amplitude. So that, coming back to the model equation (3) with the expansion (5), we first derive a system for the approximate values $p_{i}{ }^{\mathrm{III}}, A^{\mathrm{II}}$ and $B^{\mathrm{II}}$. 
We now identify the Fourier coefficients in the substitution up to $\epsilon^{4}$ for the $\theta$ independent term, to $\epsilon^{3}$ for the $\cos \theta$ and $\sin \theta$ coefficients and to $\epsilon^{2}$ for $\cos 2 \theta$ and $\sin 2 \theta$. Besides Eqs. (7), we now obtain:

$$
\begin{aligned}
& \epsilon^{2} h^{i j k l} B_{: i j k l}{ }^{0}+g^{i j} B_{i j}{ }^{I 1}=\frac{\epsilon}{2} m^{i j}\left\{\frac{A^{2}}{2}+B^{2}\right\}_{; i j}^{\mathrm{I}}, \\
& D^{I I I}-\frac{\epsilon^{2}}{2}\left\{\frac{1}{A}\left(D^{i i j} A_{; i}\right)_{; j}+2 h^{i j k l}\left(3 p_{k ; i} p_{l ; j}+4 p_{k} p_{l ; i j}\right)\right\}^{\mathrm{I}} \\
& =\epsilon\{\mathscr{T} B\}^{1 \mathrm{I}}+\frac{\epsilon^{2}}{2}\{\mathfrak{T C} C\}^{\mathrm{I}}-\frac{\epsilon^{3}}{2}\left\{\frac{1}{A} \mathscr{T}^{\mid i j}(A B)_{; i j}\right\}^{0}, \\
& \left\{D^{i i} A^{2}-\frac{\epsilon^{2}}{6}\left[D^{\mid i j k}\left(2 A A_{; j k}-A_{; j} A_{; k}\right)+\frac{1}{2}\left(D^{i j k}{ }_{; k} A^{2}\right)_{; j}\right]\right\}_{; i}^{{ }^{I I}} \\
& =\epsilon\left\{\left(\left.\mathfrak{M}\right|^{i i} A^{2}\right)_{; i}\left(B+\epsilon \frac{C}{2}\right)+\left.2 \mathfrak{M}\right|^{i i} A^{2}\left(B+\epsilon \frac{C}{2}\right)_{; i}+\mathfrak{M} A^{2} D\right\}^{1}, \\
& C^{\mathrm{I}}=\left\{\frac{\mathscr{M}}{\mathfrak{D}_{(2)}} A^{2}\right\}^{\mathrm{I}}+4 \epsilon\left\{\left(\frac{\mathscr{T}}{\mathfrak{D}_{(2)}}\right)^{2} A^{2} B\right\}^{0},
\end{aligned}
$$

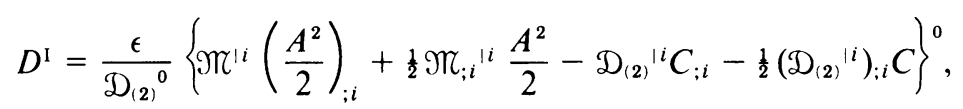

where $\mathscr{D}_{(2)}{ }^{1 i}$ means that we carry out the substitution $p_{j} \rightarrow 2 p_{j}$ in the function $\mathfrak{D}^{1 i}$.

With the expressions (76), (77) for $C^{1}$ and $D^{1}$, Eqs. (7), (73), (74) and (75) provide the required system. In addition, the approximate solution of (3) as described by (5) will be meaningful if we know the zeroth-values of the parameters $E$ and $F$ appearing in the second harmonic. These values are searched for by identifying the Fourier coefficients of $\cos 3 \theta$ and $\sin 3 \theta$. As $\mathscr{D}_{(3)}{ }^{\circ} \neq 0$, we obtain

$$
E^{0}=\frac{9}{2}\left\{\frac{\mathscr{T}^{2}}{\mathbb{D}_{(2)} \mathbb{D}_{(3)}} A^{3}\right\}^{0} \quad \text { and } \quad F^{0}=0,
$$

where $\mathfrak{D}_{(3)}$ denotes $\mathfrak{D}\left(3 p_{i}\right)$.

Coming back once again to the hypotheses of Sec. 3, with $p_{i}$ and $B$ given by Eqs. (22) and (23), we obtain from Eq. (73):

$$
g^{i j} \tilde{B}_{; i j}{ }^{\mathrm{I}}=\frac{1}{4} m^{i j}\left(A^{2}\right)_{; i j}{ }^{\mathrm{I}} .
$$

This has the same form as Eq. (24), but is now written for the first-order values of the parameters. By expanding the functions $D$ and $\mathscr{T}$ and their derivatives, and using the assumption $q_{i}=$ const., we are led, as in Sec. 3 (Eqs. (27), (28)), to the following forms for the dispersion and wave-action equations (74), (75):

$$
\begin{aligned}
& D^{i i}\left\{A \alpha_{: i}\right\}^{11}-\frac{\epsilon}{2} D^{\mid i j}\left\{A_{; i j}-A \alpha_{; i} \alpha_{; j}\right\}^{1} \\
& -\frac{\epsilon^{2}}{6} \mathfrak{D}^{\mid i j k}\left\{3 A_{; i j} \alpha_{; k}+3 A_{; i} \alpha_{; j k}+A \alpha_{; i j k}-A \alpha_{; i} \alpha_{; j} \alpha_{; k}\right\}^{0}
\end{aligned}
$$

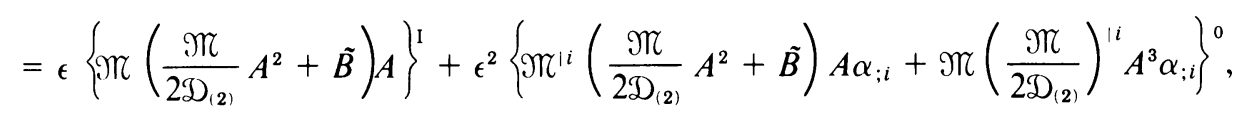




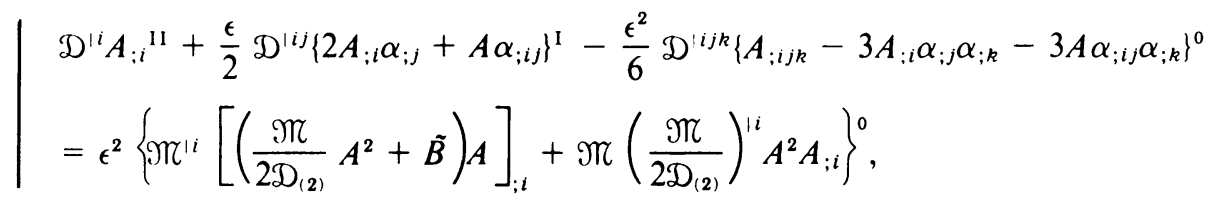

where $\mathcal{D}, \mathfrak{T}$ and their derivatives are now understood as functions of the $q_{i}$ and are therefore constant terms.

By combining these equations in the same way as in Sec. 3 and with the same definition of the complex amplitude $a$, we then obtain

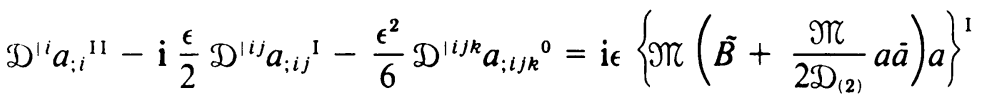

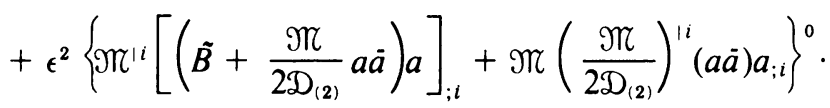

Eq. (82) together with (79), now rewritten as

$$
g^{i j} \tilde{B}_{; i j}{ }^{\mathrm{I}}=\frac{1}{4} m^{i j}(a \bar{a})_{; i j}{ }^{\mathrm{I}},
$$

form the required system for the slow variations of $a^{\mathrm{II}}$ and $\tilde{B^{\mathrm{I}}}$.

Though Eq. (83) is in the same form as Eq. (31), its integration is no longer easy, even when we suppose $\tilde{B}$ to be a function of $a$ and $\bar{a}$, since $a^{\mathrm{I}}$ is now a solution of the full equation (29), or (33) as a particular case. However, if we assume again that $\tilde{B}^{\mathrm{I}}$ is some real-valued function of $a$ and $\vec{a}$ and that the space-time is two-dimensional, we may retain the expression (32) for the value $\tilde{B}^{0}$ which is needed in the $\epsilon^{2}$ term on the right-hand side of Eq. (82). In this way, Eq. (82) may be written as

$$
\begin{aligned}
& \left(D^{1 i}+\epsilon^{2} \beta \mathbb{M}^{i i}\right) a_{; i}{ }^{11}-\mathrm{i} \frac{\epsilon}{2} D^{1 i j} a_{; i j}{ }^{\mathrm{I}}+\epsilon^{2}\left\{K^{i}(a \bar{a}) a_{; i}+L^{i}(a \bar{a})_{; i} a\right\}^{0}-\frac{\epsilon^{2}}{6} \mathscr{D}^{1 i j k} a_{: i j k}{ }^{0} \\
& =\mathrm{i} \epsilon\{\mathcal{F}(a, \bar{a}) a\}^{\mathrm{I}},
\end{aligned}
$$

where $K^{i}, L^{i}$ are known constant vectors depending on the $q_{i}, \beta$ is an integration constant to be determined by some initial condition when integrating Eq. (31), and $\mathcal{F}$ is some realvalued function of $a$ and $\bar{a}$ which may still be a linear function of $a \bar{a}$ for some particular form of the tensor $m^{i j}$. As we assume the space-time to be two-dimensional, owing to Eq. (30) for the value $a^{0}$, all the derivatives that appear in $\epsilon^{2}$ terms in Eq. (84) may be expressed as taken in the same direction.

The third derivative thus obtained, together with the nonlinear first-derivative terms, when associated with the linear first-derivative term, appear to be what would be the relevant version, for $u$ complex, of the so-called modified Korteweg-de Vries equation that reads, for $u$ real (see, e.g., [16] or [17]):

$$
u_{t}+\left(u^{3}\right)_{x}+u_{x x x}=0
$$

However, the "i-terms" in (84) keep the nonlinear (or cubic as a particular case) Schrödinger character of the equation. It must be noticed that the direction of differentiation for the linear first-derivative term is no longer the zeroth-order group direction but that it is modified by an $\epsilon^{2}$ term when $\beta \neq 0$.

It may be worth emphasizing again that the right system that governs the variations of 
$a^{11}$ and $\tilde{B^{\mathrm{I}}}$ is given by Eqs. (82), (83); or by Eqs. (29), (31) for $a^{\mathrm{I}}$ and $\tilde{B}^{0}$. However artificial the derivation of Eq. (84) may appear, it seems nevertheless interesting to see a new equation added to the arsenal of nonlinear dispersive models that have been so extensively studied in the last few years, and to show how this equation may arise.

\section{REFERENCES}

[1] G. B. Whitham, Nonlinear dispersive waves, Proc. Roy. Soc. London A 283, 238-262 (1965)

[2] G. B. Whitham, A general approach to linear and nonlinear dispersive waves using a Lagrangian, J. Fluid Mech. 22, 273-283 (1965)

[3] D. Montgomery and D. A. Tidman, Secular and non-secular behavior for the cold plasma equations, Phys. Fluids 7, 242-249 (1964)

[4] A. H. Nayfeh, Nonlinear oscillations in a hot electron plasma, Phys. Fluids 8, 1896-1898 (1965)

[5] P. A. Sturrock, Nonlinear effects in electron plasmas, Proc. Roy. Soc. London A 242, 277-299 (1957)

[6] T. Kawahara, The derivative-expansion method and nonlinear dispersive waves, J. Phys. Soc. Japan 35, 1537-1544 (1973)

[7] T. Taniuti and N. Yajima, Perturbation method for a nonlinear wave modulation, J. Math. Phys. 10, 1369-1372 (1969)

[8] T. Taniuti, Reductive perturbation method and far fields of wave equations, Prog. Theor. Phys., Supp. 55, 1-35 (1974)

[9] T. Kakutani and N. Sugimoto, Krylov-Bogoliubov-Mitropolsky method for nonlinear wave modulation, Phys. Fluids 17, 1617-1625 (1974)

[10] J. C. Luke, A perturbation method for nonlinear dispersive wave problems, Proc. Roy. Soc. London A 292, 403-412 (1966)

[11] G. B. Whitham, Two-timing, variational principles and waves, J. Fluid Mech. 44, 373-395 (1970)

[12] P. Gatignol, Sur une méthode asymptotique en théorie des ondes dispersives non-linéaires, J. Mécan. 11, 95-117 (1972)

[13] P. Gatignol, Description asymptotique des solutions d'ondes périodiques lentement variables pour l'équation de Korteweg-de Vries, C. R. Acad. Sc. Paris A 274, 1861-1864 (1972)

[14] W. D. Hayes, Conservation of action and modal wave action, Proc. Roy. Soc. London A 320, 187-208 (1970)

[15] G. B. Whitham, Linear and nonlinear waves, Part II, J. Wiley \& Sons, New York, 1974

[16] R. M. Miura, Korteweg-de Vries equation and generalizations, J. Math. Phys. 9, 1202-1204 (1968)

[17] P. Gatignol, Sur la forme et la recherche des densités invariantes associées à certaines équations aux dérivees partielles non-linéaires, Bul. Inst. Polit. Iasi 14, 51-60 (1968) 